Uwe Volker Schoch

Anspannung ist ein natürliches Phänomen und gehört zum Menschsein ebenso dazu wie Ruhe. Leben besteht aus einem ständigen Wechsel zwischen Anspannung und Entspannung. Kritisch wird es jedoch, wenn Situationen von Anspannung überhandnehmen und Möglichkeiten zum Spannungsabbau blockiert sind.

Chronischer Stress belastet im Laufe der Zeit das empfindliche Gleichgewicht, was nicht ohne Folgen bleibt. So erklären sich vegetative Herzbeschwerden, Blutdruckstörungen, Behinderungen der Atmung, Schlafstörungen, Gewichtsabnahme, psychische Labilität oder Erschöpfungszustände, um nur einige von vielen möglichen Symptomen zu nennen.

Über Bewusstsein, Sinne und Nerven kommuniziert der Mensch mit der Aussenwelt und tauscht mit ihr Informationen aus. Eine chronisch überhöhte Belastung mit Umweltreizen führt jedoch zu entsprechenden Überforderungsprozessen. Diese können sich dahingehend verselbstständigen, dass Körper und Seele dauerhaft auf das «Programm Stress» eingestellt sind, aus dem sie keinen Ausweg mehr finden.

Häufige Überlastungssituationen signalisieren dem Menschen, dass es im Moment keinen Raum gibt, um sich adäquat $\mathrm{zu}$ regenerieren und in Balance zu halten. Dieser Zustand benötigt dann eine sensible und effiziente Behandlung. Dabei kann die Psychosomatische Energetik (PSE) hilfreich sein, weil sie durch Auffinden von energetischen Blockaden und

\title{
Stress, Erschöpfung, Schlafstörungen und vegetative Labilität als Zeichen von Dysregulation
}

\author{
Bedeutung, Auswirkung und Behandlung \\ mit der Psychosomatischen Energetik
}

deren sanfte Auflösung mithilfe spezieller Homöopathika, die einen nachhaltig kräftigenden Effekt besitzen, zurück zur Gesundheit und zu einer Stabilisierung des Körper-, Geist- und Seelengleichgewichts führt.

Der Teufelskreis von Erschöpfung und mangelnder Erholung wird durchbrochen und durch aufbauende Impulse ersetzt. Im Laufe von wenigen Wochen und Monaten können sich die Funktionssysteme des belasteten Menschen nachhaltig erholen.

Die PSE, auch als sogenannter «Energiecheck» bezeichnet, deckt Energieblockaden und deren dazugehörige seelische Themen auf, um diese mit individuell ausgetesteten Arzneimitteln nach und nach abschmelzen zu lassen. Dadurch bekommen Körper und Seele Impulse und können sich neu strukturieren, sodass die Heilwerdung eintreten kann.

Aus meiner langjährigen Erfahrung als Heilpraktiker und Zertifizierter Energietherapeut kann ich von vielen positiv verlaufenden Fällen in meiner naturheilkundlichen Praxis berichten. Zur Veranschaulichung und Vorgehensweise möchte ich nun einen Fall schildern.

\section{Fallbericht}

Ein Mann Mitte 50 kommt wegen Erschöpfung und Schlafstörungen in meine Praxis. In der Anamnese erzählt er mir, dass er sich in seinem Beruf als Manager einer grossen Wer- befirma total überfordert fühle, völlig kaputt sei, sich kaum mehr konzentrieren könne und trotz der körperlichen Erschöpfung schlecht schlafe.

Von Zeit zu Zeit habe er Schwindelanfälle, Herzrasen und Panikattacken, lässt er mich weiter wissen, und dass er ab und an Angst verspüre, seiner Aufgabe nicht mehr gewachsen zu sein, ihm nichts mehr richtig Spass mache, manchmal alles so sinnlos erscheine. Er sei am Limit angekommen und wolle jetzt, nachdem schulmedizinisch keinerlei Befund diagnostiziert werden konnte, einen anderen Weg gehen, weil er wisse, dass er diesem Teufelskreis entkommen will.

Ich vermute, dass er kurz vor einem Burnout steht oder schon mittendrin ist und es höchste Zeit wird, etwas zu unternehmen, sodass er zurück zu seiner Lebensfreude und Energie findet, die ihm mittlerweile abhandengekommen ist. Dazu benötige ich zunächst einmal seine Energiewerte, die mithilfe eines speziellen Gerätes und dem Armlängenreflextest nach Assche, einem kinesiologischen Test, ermittelt werden.

Es verwundert mich nach seinen Schilderungen nicht, dass sein Vitalwert, der die körperliche Fitness darstellt, ziemlich schlecht ist, wie auch sein Emotionalwert, der mit dem seelischen Gleichgewicht, der guten Laune, der Lebensfreude sowie dem Antrieb zu tun hat, und sein Mentalwert, d.h. sein Denkapparat, der für die Alltagstauglichkeit zuständig ist. Die Werte spiegeln sein Empfinden und seine Aussagen völlig wider.

\section{KARGER}

Fax +49761 4520714 Information@Karger.d www.karger.com
(๑) 2012 S. Karger GmbH, Freiburg

Accessible online at: www.karger.com/szg
Uwe Volker Schoch

Praxis für ganzheitliche Heilweisen

Schulstrasse 2, 78337 Öhningen-Schienen, Deutschland

Tel. +497735919869

schoch-uwe@t-online.de

www.naturheilpraxis-uwe-schoch.de 
Der Körper zeigt eine Blockade im Zentrum des Unterbauchs auf, wo es um die Entwicklung von Mut und Kraft in der Auseinandersetzung mit Stress und bedrohlichen Situationen geht. Ich und die Welt - Eigenfindung ist mitunter angesagt. Das dazugehörige seelische Thema heisst Durchhalten, somatisierte Ängste, d.h. Ängste, die in den Körper gehen. Selbstbeherrscht sein wollen trotz des Gefühls der Hilflosigkeit steht in seinem Programm. Überforderung und Durchhalten fast um jeden Preis hat er sich auf die Fahne geschrieben.

Als Akutmittel fungiert Anxiovita $^{\circledR}$, das hilfreich bei allen Formen von Angst, Panik und Unruhezuständen ist sowie Stress im Körper signalisiert und abbauen hilft. Des Weiteren ist Paravita ${ }^{\circledR}$ angezeigt, das Verkrampfungen löst und Erschöpfungszustände, die nach langem Stress auftreten, bessert.

Als ich ihm all das Gefundene erzähle, staunt er, kann sich aber ganz und gar damit identifizieren. Es geht also darum, das Gefühl zu haben, keine innere Stärke mehr zu besitzen und dass der Körper angstvoll reagiert, der Kopf aber stark sein möchte. Die Fähigkeit zu entwickeln, eigene Grenzen anzuerkennen und Erholung $\mathrm{zu}$ suchen, ist eine Lernaufgabe für ihn. Inneren Stress zu überwinden als auch Vertrauen zu entwickeln, dass in Ruhe alles leichter geht, sollte ihm bewusst werden, damit er dies transformieren kann.

Als Hausaufgabe bekommt er zusätzlich zur Tropfeneinnahme den Lösungssatz mit, der im Sinne einer
Wegweisung gesehen und benutzt werden kann, um seine Eigenverantwortung zu stärken: Ich weiss, dass mein Körper seine eigene innere Weisheit hat, in der ich sicher aufgehoben bin, oder anders ausgedrückt: Ich lerne, dass ich tief in mir weiss, was gut für mich ist. Mein Kopf und mein Körper sind sich einig. Des Weiteren bitte ich ihn in unserem Gespräch, einmal nachzudenken, ob und, wenn ja, was er auf seiner Arbeit verändern kann. Er ist 3 Monate beschäftigt, diese Blockade aufzulösen, und wird danach erneut vorstellig bei mir.

In der Zweitkonsultation erfahre ich, dass zwischenzeitlich eine Umwandlung zum Positiven hin geschehen ist und er die Stelle innerhalb der Firma gewechselt hat. Er spüre zwar noch Stress, aber lange nicht mehr so oft, und könne gelassener damit umgehen. Seine Beschwerden haben sich wesentlich gebessert, er kann sich wieder besser konzentrieren, sein Schlaf ist erholsamer, sein Herzrasen tritt nur noch selten auf, die Schwindelanfälle sind wesentlich weniger geworden und seine Panikattacken sowie die Ängste haben sich auf ein Minimum reduziert.

Er wisse, dass das natürlich noch nicht das Ende der Behandlung sei, und will dranbleiben, um weitere Energieblockaden mit den dazugehörigen seelischen Themen anzugehen, sodass sein Körper wieder selbstständig regulieren kann und er zurück zu seinem Gleichgewicht kommt. Nach ca. 1 Jahr fühlt er sich wie neu geboren, hat eine unbändige Energie, kann verschiedene Dinge, sei es im Privaten oder Beruflichen, viel gelassener sehen, ist einfach freier und gelöster geworden.

\section{Schlussfolgerung}

Aus diesem und anderen ähnlich gelagerten Fällen kann ich aus langjähriger Praxiserfahrung folgendes Fazit ziehen:

Die PSE ist eine denkbar einfache, ganzheitliche und kostengünstige Therapiemethode, bei der Energiedefizite mit entsprechenden seelischen Themen selbst nach Jahren oder gar Jahrzehnten mühelos aufgedeckt werden können, um diese mit entsprechenden homöopathischen Komplexmitteln sanft zu beheben. Durch die Auflösung energetischer Blockaden kann der Patient aus dem Teufelskreis von Stress, Hektik und Nervosität aussteigen, um danach gestärkt, zufrieden, freudvoll und authentisch daraus hervorzugehen.

Meine Aufgabe ist es, ihm zu vermitteln, dass er die Verantwortung für sich selbst trägt, ich ihn zwar begleiten, den Erfolg aber nicht für ihn «machen» kann. Zuwendung, Vermittlung von Freude, Zuversicht, Hoffnung sowie die Impulse der Mittel können Heilprozesse in Gang setzen und sind ein wichtiger Bestandteil auf dem Weg zur Gesundung. Nur wenn uns genügend Lebensenergie durchströmt, dann fühlen wir uns wohl, arbeitet unser Immunsystem ungestört und sind wir ausgeglichen. 